\title{
ALADDIN: an optimized ground-based precursor for DARWIN
}

\author{
Vincent Coudé du Foresto ${ }^{1}$, Olivier Absil ${ }^{2}$, \\ Marc Barillot ${ }^{3}$, and Mark Swain ${ }^{4}$ \\ ${ }^{1}$ LESIA, Observatoire de Paris, 92190 Meudon, France \\ email: vincent.foresto@obspm.fr \\ ${ }^{2}$ Institut d'Astrophysique et de Géophysique, Université de Liège, 4000 Sart-Tilman, Belgium \\ email: absil@astro.ulg.ac.be \\ ${ }^{3}$ Alcatel Alenia Space, 06150 Cannes, France \\ email: marc.barillot@alcatelaleniaspace.com \\ ${ }^{4}$ Max-Planck Institut fur Astronomie, 69117 Heidelberg, Germany \\ email: swain@mpia-hd.mpg.de
}

\begin{abstract}
The ALADDIN concept is an integrated Antarctic-based L-band experiment whose purpose is to demonstrate nulling interferometry and to prepare the DARWIN mission. Because of their privileged location, the relatively modest collectors $(1 \mathrm{~m})$ and baseline (up to $40 \mathrm{~m}$ ) are sufficient to achieve a sensitivity (in terms of detectable zodi levels) which is about twice better than that of a nulling instrument on a large interferometer (such as GENIE at the VLTI), and to reach the 20-zodi threshold value identified to carry out the DARWIN precursor science. These numbers are based on a preliminary design study by Alcatel Alenia Space and were obtained using the same simulation software as the one employed for GENIE. The integrated design enables top-level optimization and full access to the light collectors for the duration of the experiment, while reducing the complexity of the nulling breadboard.
\end{abstract}

Keywords. Instrumentation: interferometers, antarctic astronomy, techniques: interferometric, planetary systems, protoplanetary disks.

\section{Introduction}

The DARWIN mission considered by the European Space Agency (detection and characterization of habitable planets around nearby stars using interferometric nulling (Léger et al. (1996)) will require a ground-based precursor, whose main objectives are to validate on-sky the technique of nulling interferometry, as well as to carry out scientific observations in preparation of the space mission. Most notably the amount of dust around DARWIN targets needs to be investigated as high levels of exozodiacal light (about 20 times, or more, the amount of the solar level), or an asymmetric distribution of dust, may hamper the detection of Earth-like planets.

The first option for a ground-based precursor is to build an instrument designed for an existing interferometer, such as the GENIE L-band nulling instrument which is being studied for the VLTI (den Hartog et al. (2005)). This ties the design and performance of the instrument to those of the suppporting infrastructure. A radically different approach, presented here, is the search for the optimal system at the optimal site in order to achieve the ultimate performance available from the ground.

More specifically, it appears that four factors are key to the success of a ground-based precursor of DARWIN:

- The breadboard should be designed in such a way that its concept focuses on issues relevant to the DARWIN mission (nulling, phase stabilization, data analysis), avoiding 
as much as possible design and engineering challenges which are circumstancial to the demonstration experiment (such as interfaces to an outside system, control loops not needed in DARWIN etc.);

- Because of the complex nature of an interferometer and the many real time interactions between its separate elements, the ultimate performance can only be achieved if the design is optimized at the system level;

- The quality of the site is critical and drives the final performance of the system. Therefore the experiment has to be located on the best accessible ground-based site. As for any interferometer subject to atmospheric turbulence, the largest possible seeing cell size is required to maximize the number of coherent photons. But most importantly, turbulence needs to be slow as it relaxes the requirements on the control loops and enables lower residuals. Finally, for efficient nulling in the infrared a low and stable background is required;

- The nulling demonstrator is a pathfinder mission and should be conceived as such, integrating development, deployment and operations (for a well defined time frame) into the concept.

The ALADDIN (Antarctic L-band Astrophysics Discovery Demonstrator for Interferometric Nulling) concept results from these considerations.

\section{The concept}

\subsection{Site selection}

It has been realized in the last few years that the high antarctic plateau, and more specifically the Dome $\mathrm{C}$ site (at an elevation $3250 \mathrm{~m}$ and $-75 \mathrm{deg}$ latitude) provides a place of strong potential for several types of astronomical observations, and most notably for infrared interferometry. This is due to a combination of several unique features, among which:

- Low environmental and sky temperatures (195-235 K) which result in a low and stable emission from the thermal background;

- An extremely dry air $(250 \mu \mathrm{m}$ precipitable water vapor typical) which results in improved infrared transmission, reduced dispersion variations and extended atmospheric windows (for example from 2.8 to $4.2 \mu \mathrm{m}$ around the $\mathrm{L}$ band);

- Low surface winds and low free air turbulence, with a quasi-absence of jet streams as Dome $\mathrm{C}$ is located inside the polar vortex.

The dark time atmosphere has been investigated by different techniques (Lawrence et al. (2003), Agabi et al. (2006)). Most of the turbulence is concentrated in the ground layer (whose typical height is $30 \mathrm{~m}$ ), above which the seeing has been found to be much lower than at any other measured site ( 0.27 arcsec median value). Besides, long coherence times ( $\geqslant 7 \mathrm{~ms}$ in normalized conditions) have strong benefits for the performance (lower residuals) of real time control loops (tip-tilt, fringe tracking). The Dome $\mathrm{C}$ site is accessible with logistics developed by the French (IPEV) and Italian (PNRA) polar institutes who built the Concordia station there. Most humans reach the site by plane, while heavy equipment is shipped in standard containers by boat to the coast and then by "traverses" (pack trains of Caterpillar trucks) onto the glacier slopes up to Dome C, which thus provides a near space quality environment for infrared interferometry at a small fraction of the access cost.

\subsection{Design}

ALADDIN is conceived to optimize nulling interferometry performance and focus on issues relevant to the DARWIN mission (nulling, phase stabilization, data analysis), 
avoiding as much as possible engineering challenges which are circumstancial to the demonstration experiment (such as interfaces to an outside system, control loops not needed in DARWIN etc...).

The main features of a preliminary optomechanical design (Figure 1) studied by Alcatel Alenia Space are:

- A pair of $1 \mathrm{~m}$ collectors (siderostats) located above the ground layer, which can move on a circular path to maintain the source at the meridian of the baseline. Incidence angles are nearly constant during observations enabling better polarization control;

- An adjustable baseline (4-40 m), optimized for each source to obtain the best compromise between angular resolution and stellar leakage. The baseline can be reverted for calibration purposes;

- A streamlined optical train (off-axis telescopes and only 9 warm surfaces from the sky to the entrance of the bench, vs. 21 for the VLTI) for enhanced throughput and overall emissivity;

- A fixed, cryogenized bench with a single Bracewell nulling beam combiner;

- K-band control and L-band science. Thanks to the dry atmosphere no dispersion control loop is needed. The intensity control loop can also avoided (only a monitoring is needed) due to low $d / r_{0}$ ratio. Because of the longer coherence time of the turbulence, tip-tilt and fringe tracking loop rates are considerably reduced (down to a few $\mathrm{kHz}$ ). A preliminary analysis shows that, contrary to intuitive expectations, the cost and schedule of ALADDIN could be comparable to those of GENIE, as the effort to build the platform is balanced by the many simplifications and optimizations brought by the integrated design and the Antarctic location. The development plan can be made more robust as it can be parallelized and the reliance on the nominal performance of an external platform disappears. The integrated design enables a prior implementation in a temperate site, with full functional testing, before deployment at Dome C.

\section{Performance}

The science performance of ALADDIN can be evaluated using the simulation software package "GENIEsim" developed to analyze GENIE (Absil et al. (2005)), substituting as input the preliminary design shown here and the Dome $\mathrm{C}$ atmospheric properties above the ground layer as measured by Lawrence et al. (2003). This protocol enables a direct comparison between the two systems (see Table 1) for a similar integration time (in this case $1800 \mathrm{~s}$ ).

The quality factor retained for science performance is the $5 \sigma$ detectivity of exozodiacal light. It is affected by several factors and most notably the instrumental leakage control (thermal background and phase stabilization), the residual shot noise from the (not fully nulled) stellar light, and the calibration of stellar leakage (due to the angular extension of the stellar disk).

Thanks to the quality of the site and its system-level optimization, even with $1 \mathrm{~m}$ collectors the sensitivity of ALADDIN is always better than the sensitivity of GENIE at the VLTI. As can be expected, the gain is largest (up to a factor 2.9 for a K0V star at $5 \mathrm{pc}$ ) for nearby stars which benefit most from the adjustable baseline.

\section{Conclusion}

It appears that the primary objectives of a DARWIN ground-based precursor can be better served by an integrated experiment located on the Antarctic plateau, which would provide both a more relevant technical demonstration and better preparatory science. 


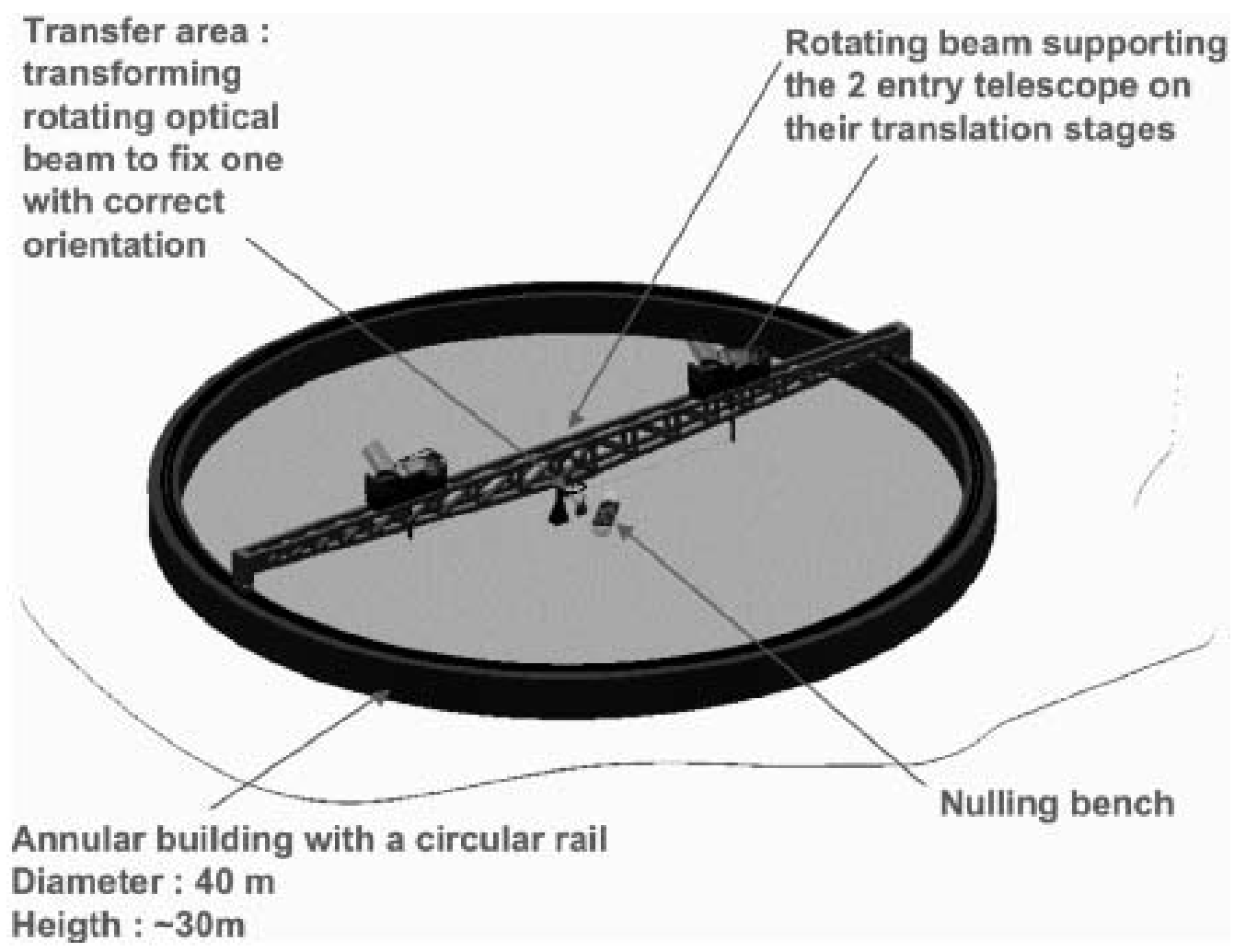

Figure 1. ALADDIN preliminary optomechanical concept.

Table 1. Comparative performance of GENIE and ALADDIN (in units of solar zodiacal light, lower is better) for the detection of exozodiacal light around four fiducial DARWIN targets, assuming a disk diameter known to within $0.5 \%$ for the calibration of stellar leakage. GENIE/VLTI values are derived from ESA's phase A study (den Hartog et al. (2005)) and are shown for the pair of collectors (UTs $(8 \mathrm{~m})$ or ATs $(1.8 \mathrm{~m})$ that provide the best result in each case.

\begin{tabular}{lccc}
\hline Source & GENIE/VLTI & ALADDIN & Detectivity ratio \\
\hline K0V at $5 \mathrm{pc}$ & $110(\mathrm{ATs})$ & 38 & 2.9 \\
G5V at $10 \mathrm{pc}$ & 56 (UTs) & 26 & 2.2 \\
G0V at $20 \mathrm{pc}$ & 37 (UTs) & 20 & 1.9 \\
G0V at $30 \mathrm{pc}$ & 48 (UTs) & 23 & 2.1
\end{tabular}

While many issues (including critical ones) remain to be investigated, it is worthwhile to further explore this option.

\section{References}

Absil, O., den Hartog, R., et al. 2005 A $\mathscr{E} A$, in press.

Agabi, A., Aristidi, E., Azouit, M., Fossat, E., Martin, F., Sadibekova, T., Vernin, J., \& Ziad, A. $2006 P A S P$, in press.

den Hartog, R., et al., these Proceedings.

Lawrence, J. S., Ashley, M. C. B., Tokovinin, A., \& Travouillon, T. 2003, Nature 431, 278

Leger, A., Mariotti, J. M., Mennesson, B., Ollivier, M., Puget, J. L., Rouan, D., \& Schneider, J. 1996, Icarus 123, 249 